\title{
Nabla discrete fractional Grüss type inequality
}

\author{
A Feza Güvenilir ${ }^{1}$, Billur Kaymakçalan ${ }^{2 *}$, Allan C Peterson ${ }^{3}$ and Kenan Taş²
}

\section{"Correspondence:}

billur@cankaya.edu.tr

${ }^{2}$ Department of Mathematics and

Computer Science, Çankaya

University, Ankara, 06810, Turkey

Full list of author information is

available at the end of the article

\begin{abstract}
Properties of the discrete fractional calculus in the sense of a backward difference are introduced and developed. Here, we prove a more general version of the Grüss type inequality for the nabla fractional case. An example of our main result is given.
\end{abstract}

MSC: Primary 39A12; 34A25; 26A33; secondary 26D15; 26D20

Keywords: nabla discrete fractional calculus; nabla discrete Grüss inequality

\section{Introduction}

The Grüss inequality is of great interest in differential and difference equations as well as many other areas of mathematics [1-5]. The classical inequality was proved by Grüss in 1935 [3]: if $f$ and $g$ are continuous functions on $[a, b]$ satisfying $\varphi \leq f(t) \leq \Phi$ and $\gamma \leq$ $g(t) \leq \Gamma$ for all $t \in[a, b]$, then

$$
\left|\frac{1}{b-a} \int_{a}^{b} f(t) g(t) d t-\frac{1}{(b-a)^{2}} \int_{a}^{b} f(t) d t \int_{a}^{b} g(t) d t\right| \leq \frac{1}{4}(\Phi-\varphi)(\Gamma-\gamma)
$$

The literature on Grüss type inequalities is extensive, and many extensions of the classical inequality (1) have been intensively studied by many authors in the 21st century [6-12]. Here we are interested in Grüss type inequalities in the nabla fractional calculus case.

We begin with basic definitions and notation from the nabla calculus that are used in this paper. The delta calculus analog has been studied in detail, and a general overview is given in [9]. The domains used in this paper are denoted by $\mathbb{N}_{a}$ where $a \in \mathbb{R}$. This is a discrete time scale with a graininess of 1 , so it is defined as

$$
\mathbb{N}_{a}:=\{a, a+1, a+2, \ldots\}
$$

For $\mathbb{N}_{a}$, we use the terminology that a function's domain, in the case studied here, is based at $a$. We use the $\rho$-function, or backward jump function, from the time scale as $\rho: \mathbb{N}_{a} \rightarrow$ $\mathbb{N}_{a}$, given by $\rho(t):=\max \{a, t-1\}$. We define the backward difference operator, or the nabla operator $(\nabla)$, for a function $f: \mathbb{N}_{a} \rightarrow \mathbb{R}$ by

$$
(\nabla f)(t):=f(t)-f(\rho(t))=f(t)-f(t-1) .
$$


In this paper, we use the convention that

$$
\nabla f(t)=(\nabla f)(t)
$$

We then define higher order differences recursively by

$$
\nabla^{n} f(t):=\nabla\left(\nabla^{n-1} f(t)\right)
$$

for $t \in \mathbb{N}_{a+n}$ where $n \in \mathbb{N}$. We take as convention that $\nabla^{0}$ is the identity operator.

Based on these preliminary definitions, we say $F$ is an anti-nabla difference of $f$ on $\mathbb{N}_{a}$ if and only if $\nabla F(t)=f(t)$ for $t \in \mathbb{N}_{a+1}$. We then define the definite nabla integral of $f: \mathbb{N}_{a} \rightarrow \mathbb{R}$ by

$$
\int_{c}^{d} f(t) \nabla t:= \begin{cases}\sum_{t=c+1}^{d} f(t), & \text { if } c<d, \\ 0, & \text { if } c=d, \\ -\sum_{t=d+1}^{c} f(t), & \text { if } d<c,\end{cases}
$$

where $c, d \in \mathbb{N}_{a}$. We now give the fundamental theorem of nabla calculus.

Theorem 1.1 (Fundamental theorem of nabla calculus) Let $f: \mathbb{N}_{a} \rightarrow \mathbb{R}$ and let $F$ be an anti-nabla difference off on $\mathbb{N}_{a}$, then for any $c, d \in \mathbb{N}_{a}$ we have

$$
\int_{c}^{d} f(t) \nabla t=F(d)-F(c)
$$

The nabla product rule for two functions $u, v: \mathbb{N}_{a} \rightarrow \mathbb{R}$ and $t \in \mathbb{N}_{a+1}$ is given by

$$
\nabla(u(t) v(t))=u(t) \nabla v(t)+v(\rho(t)) \nabla u(t) .
$$

This immediately leads to the summation by parts formula for the nabla calculus:

$$
\sum_{s=b+1}^{c} u(t) \nabla v(t)=\left.u(t) v(t)\right|_{b} ^{c}-\sum_{s=b+1}^{c} v(\rho(t)) \nabla u(t)
$$

Discrete fractional initial value problems have been intensively studied by many authors. Some of the very recent results are in [13-15].

Now that we have established the basic definitions of the nabla calculus, we will move on to extending these definitions to the fractional case and establish definitions for the fractional sum and fractional difference which are analogues to the continuous fractional derivative.

In order to do this, we remind the reader of the rising factorial function. For $n, t \in \mathbb{N}$, the rising factorial function is defined by

$$
t^{\bar{n}}:=t(t+1) \cdots(t+n-1)=\frac{(t+n-1) !}{(t-1) !} .
$$

This definition can be extended for fractional values using the gamma function as follows. 
Definition 1 (Rising function) For $t, \alpha \in \mathbb{R}$, the rising function is defined by

$$
t^{\bar{\alpha}}:=\frac{\Gamma(t+\alpha)}{\Gamma(t)} .
$$

To motivate the definition of a fractional sum, we look at the definition of the integral sum derived from the repeated summation rule.

Definition 2 For any given positive real number $\alpha$, the (nabla) left fractional sum of order $\alpha>0$ is defined by

$$
\nabla_{a}^{-\alpha} f(t)=\frac{1}{\Gamma(\alpha)} \sum_{s=a+1}^{t}(t-\rho(s))^{\overline{\alpha-1}} f(s)
$$

where $t \in\{a+1, a+2, \ldots\}$, and

$$
\nabla_{a}^{-\alpha} f(t)=\frac{1}{\Gamma(\alpha)} \sum_{s=a}^{t}(t-\rho(s))^{\overline{\alpha-1}} f(s)
$$

where $t \in\{a, a+1, a+2, \ldots\}$.

Theorem 1.2 ([16]) Let $f$ be a real-valued function defined on $\mathbb{N}_{a}$ and $\alpha, \beta>0$. Then

$$
\nabla_{a}^{-\alpha} \nabla_{a}^{-\beta} f(t)=\nabla_{a}^{-(\alpha+\beta)} f(t)=\nabla_{a}^{-\beta} \nabla_{a}^{-\alpha} f(t)
$$

Definition 3 (Fractional Caputo like nabla difference) For $\mu>0, m-1<\mu<m, m=\lceil\mu\rceil$ (where $\lceil\cdot\rceil$ is the ceiling function), $m \in \mathbb{N}, v=m-\mu$, we have the following:

$$
\nabla_{a *}^{\mu} f(t)=\nabla_{a}^{-v}\left(\nabla^{m} f(t)\right), \quad t \in \mathbb{N}_{a} .
$$

We also will use the following discrete Taylor formula.

Theorem 1.3 ([17]) Let $f: \mathbb{Z} \rightarrow \mathbb{R}$ be a function and let $a \in \mathbb{Z}$. Then, for all $t \in \mathbb{Z}$ with $t \in \mathbb{N}_{a+m}$ we have the representation

$$
f(t)=\sum_{k=0}^{m-1} \frac{(t-a)^{\bar{k}}}{k !} \nabla^{k} f(a)+\frac{1}{(m-1) !} \sum_{\tau=a+1}^{t}(t-\tau+1)^{\overline{m-1}} \nabla^{m} f(\tau) .
$$

The following discrete backward fractional Taylor formula will be useful.

Theorem 1.4 ([18]) Let $f: \mathbb{Z} \rightarrow \mathbb{R}$ be a function and let $a \in \mathbb{Z}$. Here $m-1<\mu<m, m=\lceil\mu\rceil$, $\mu>0$. Then for all $t \in \mathbb{N}_{a+m}$ we have the representation

$$
f(t)=\sum_{k=0}^{m-1} \frac{(t-a)^{\bar{k}}}{k !} \nabla^{k} f(a)+\frac{1}{\Gamma(\mu)} \sum_{\tau=a+1}^{t}(t-\tau+1)^{\overline{\mu-1}} \nabla_{(a+1) *}^{\mu} f(\tau) .
$$


Corollary 1.1 (To Theorem 1.4) Additionally assume that $\nabla^{k} f(a)=0$, for $k=0,1,2, \ldots$, $m-1$. Then

$$
f(t)=\frac{1}{\Gamma(\mu)} \sum_{\tau=a+1}^{t}(t-\tau+1)^{\overline{\mu-1}} \nabla_{(a+1) *}^{\mu} f(\tau), \quad \forall t \geq a+m .
$$

Also, we have the following.

Theorem $1.5([18])$ Let $p \in \mathbb{N}, v>p, a \in \mathbb{N}$. Then

$$
\nabla^{p}\left(\nabla_{a}^{-v} f(t)\right)=\nabla_{a}^{-(v-p)} f(t)
$$

for $t \in \mathbb{N}_{a}$.

Now, we give the following discrete backward fractional extended Taylor formula.

Theorem 1.6 ([18]) Let $f: \mathbb{Z} \rightarrow \mathbb{R}$ be a function and let $a \in \mathbb{Z}_{+}$. Here $m-1<\mu<m, m=$ $\lceil\mu\rceil, \mu>0$. Consider $p \in \mathbb{N}: \mu>p$. Then for all $t \geq a+m, t \in \mathbb{N}$, we have the representation

$$
\nabla^{p} f(t)=\sum_{k=p}^{m-1} \frac{(t-a)^{\overline{k-p}}}{(k-p) !} \nabla^{k} f(a)+\frac{1}{\Gamma(\mu-p)} \sum_{\tau=a+1}^{t}(t-\tau+1)^{\overline{\mu-p-1}} \nabla_{(a+1) *}^{\mu} f(\tau)
$$

Corollary 1.2 (To Theorem 1.6) Additionally assume that $\nabla^{k} f(a)=0$, for $k=p, \ldots, m-1$.

Then

$$
\nabla^{p} f(t)=\frac{1}{\Gamma(\mu-p)} \sum_{\tau=a+1}^{t}(t-\tau+1)^{\overline{\mu-p-1}} \nabla_{(a+1) *}^{\mu} f(\tau), \quad \forall t \geq a+m, t \in \mathbb{N} .
$$

Remark 1.1 Let $f$ be defined on $\{a-m+1, a-m+2, \ldots, b\}$, where $b-a$ is an integer. Then (3) and (6) are valid only for $t \in\{a+m, \ldots, b\}$. Here we must assume that $a+m<b$.

\section{Main results}

We present the following discrete nabla Grüss type inequality.

Theorem 2.1 Let $m-1<\mu<m, m=\lceil\mu\rceil$ non-integer, $\mu>0 ; p, a \in \mathbb{Z}_{+}$with $\mu>p$. Consider $b \in \mathbb{N}$ such that $a+m<b$. Let $f$ and $g$ two real-valued functions defined on $\{a-m+1, a-$ $m+2, \ldots, b\}$. Here $j \in\{a+m, \ldots, b\}$. Assume that $\nabla^{k} f(a)=\nabla^{k} g(a)=0$, for $k=p+1, \ldots, m-1$ and

$$
m_{1} \leq \nabla_{(a+1) *}^{\mu} f(s) \leq M_{1}, \quad m_{2} \leq \nabla_{(a+1) *}^{\mu} g(s) \leq M_{2}
$$

for $s \in\{a+1, \ldots, b\}$, then

$$
\begin{aligned}
& \frac{1}{b-a-m} \sum_{j=a+m+1}^{b}\left[\nabla^{p} f(j) \nabla^{p} g(j)\right]-\frac{1}{(b-a-m)^{2}}\left(\sum_{j=a+m+1}^{b} \nabla^{p} f(j)\right)\left(\sum_{j=a+m+1}^{b} \nabla^{p} g(j)\right) \\
& <\frac{M_{1} M_{2} C_{1}-m_{1} m_{2} C_{2}}{(b-a-m)^{2}[\Gamma(\mu-p+1)]^{2}},
\end{aligned}
$$


where $m_{1}, m_{2}, M_{1}$, and $M_{2}$ are positive constants, and

$$
\begin{aligned}
& C_{1}:=\left[Q(b-a-m)(b-a-m+4)^{\frac{1}{2}}+(b-a-m)^{2}\left[2^{\overline{\mu-p}}\right]^{2}\right], \\
& C_{2}:=\left[\frac{(b-a+1)^{\overline{\mu-p+1}}-(m)^{\overline{\mu-p+1}}}{\mu-p+1}-(b-a-m) \Gamma(\mu-p+2)\right]^{2}, \\
& Q:=\left(\sum_{s=m+3}^{b-a+2}\left[(s)^{\overline{\mu-p}}\right]^{4}\right)^{\frac{1}{2}} .
\end{aligned}
$$

Proof By Theorem 1.6, we have

$$
\begin{aligned}
& \nabla^{p} f(j)=\nabla^{p} f(a)+\frac{1}{\Gamma(\mu-p)}\left[\sum_{\tau=a+1}^{j}(j-\tau+1)^{\overline{\mu-p-1}}\left(\nabla_{(a+1) *}^{\mu} f(\tau)\right)\right], \\
& \nabla^{p} g(j)=\nabla^{p} g(a)+\frac{1}{\Gamma(\mu-p)}\left[\sum_{\tau=a+1}^{j}(j-\tau+1)^{\overline{\mu-p-1}}\left(\nabla_{(a+1) *}^{\mu} g(\tau)\right)\right]
\end{aligned}
$$

for $j \in\{a+m+1, a+m+2, \ldots, b\}$.

We get

$$
\begin{aligned}
\left(\nabla^{p} f(j)\right)\left(\nabla^{p} g(j)\right)= & \frac{1}{[\Gamma(\mu-p)]^{2}}\left[\sum_{\tau=a+1}^{j}(j-\tau+1)^{\overline{\mu-p-1}} \nabla_{(a+1) *}^{\mu} f(\tau)\right] \\
& \cdot\left[\sum_{\tau=a+1}^{j}(j-\tau+1)^{\overline{\mu-p-1}} \nabla_{(a+1) *}^{\mu} g(\tau)\right] \\
& +\frac{\nabla^{p} f(a)}{\Gamma(\mu-p)}\left[\sum_{\tau=a+1}^{j}(j-\tau+1)^{\overline{\mu-p-1}} \nabla_{(a+1) *}^{\mu} g(\tau)\right] \\
& +\frac{\nabla^{p} g(a)}{\Gamma(\mu-p)}\left[\sum_{\tau=a+1}^{j}(j-\tau+1)^{\overline{\mu-p-1}} \nabla_{(a+1) *}^{\mu} f(\tau)\right] \\
& +\left(\nabla^{p} f(a)\right)\left(\nabla^{p} g(a)\right) .
\end{aligned}
$$

If we take the sum from $a+m+1$ to $b$ we get

$$
\begin{aligned}
\sum_{j=a+m+1}^{b}\left[\left(\nabla^{p} f(j)\right)\left(\nabla^{p} g(j)\right)\right]= & \left.\nabla^{p} f(a)\right)\left(\nabla^{p} g(a)\right) \\
& +\frac{\nabla^{p} f(a)}{\Gamma(\mu-p)}\left\{\sum_{j=a+m+1}^{b}\left[\sum_{\tau=a+1}^{j}(j-\tau+1)^{\overline{\mu-p-1}}\left(\nabla_{(a+1) *}^{\mu} g(\tau)\right)\right]\right\} \\
& +\frac{\nabla^{p} g(a)}{\Gamma(\mu-p)}\left\{\sum_{j=a+m+1}^{b}\left[\sum_{\tau=a+1}^{j}(j-\tau+1)^{\overline{\mu-p-1}}\left(\nabla_{(a+1) *}^{\mu} f(\tau)\right)\right]\right\} \\
& +\frac{1}{[\Gamma(\mu-p)]^{2}} \sum_{j=a+m+1}^{b}\left\{\left[\sum_{\tau=a+1}^{j}(j-\tau+1)^{\overline{\mu-p-1}}\left(\nabla_{(a+1) *}^{\mu} f(\tau)\right)\right]\right. \\
& \left.\cdot\left[\sum_{\tau=a+1}^{j}(j-\tau+1)^{\overline{\mu-p-1}}\left(\nabla_{(a+1) *}^{\mu} g(\tau)\right)\right]\right\} .
\end{aligned}
$$


Then

$$
\begin{aligned}
\frac{1}{b-a}-m & \sum_{j=a+m+1}^{b}\left[\left(\nabla^{p} f(j)\right)\left(\nabla^{p} g(j)\right)\right] \\
= & \frac{1}{b-a-m}\left(\nabla^{p} f(a)\right)\left(\nabla^{p} g(a)\right) \\
& +\frac{1}{(b-a-m)[\Gamma(\mu-p)]^{2}} \sum_{j=a+m+1}^{b}\left\{\left[\sum_{\tau=a+1}^{j}(j-\tau+1)^{\overline{\mu-p-1}}\left(\nabla_{(a+1) *}^{\mu} f(\tau)\right)\right]\right. \\
& \left.+\left[\sum_{\tau=a+1}^{j}(j-\tau+1)^{\overline{\mu-p-1}}\left(\nabla_{(a+1) *}^{\mu} g(\tau)\right)\right]\right\} \\
& +\frac{\nabla^{p} f(a)}{(b-a-m) \Gamma(\mu-p)}\left\{\sum_{j=a+m+1}^{b}\left[\sum_{\tau=a+1}^{j}(j-\tau+1)^{\overline{\mu-p-1}}\left(\nabla_{(a+1) *}^{\mu} g(\tau)\right)\right]\right\} \\
& +\frac{\nabla^{p} g(a)}{(b-a-m) \Gamma(\mu-p)}\left\{\sum_{j=a+m+1}^{b}\left[\sum_{\tau=a+1}^{j}(j-\tau+1)^{\overline{\mu-p-1}}\left(\nabla_{(a+1) *}^{\mu} f(\tau)\right)\right]\right\} .
\end{aligned}
$$

On the other hand,

$$
\begin{aligned}
& \frac{1}{b-a-m}\left[\sum_{j=a+m+1}^{b}\left(\nabla^{p} f(j)\right)\right] \\
& =\nabla^{p} f(a) \\
& \quad+\frac{1}{(b-a-m) \Gamma(\mu-p)} \sum_{j=a+m+1}^{b}\left(\sum_{\tau=a+1}^{j}(j-\tau+1)^{\overline{\mu-p-1}}\left(\nabla_{(a+1) *}^{\mu} f(\tau)\right)\right)
\end{aligned}
$$

and

$$
\begin{aligned}
& \frac{1}{b-a-m}\left[\sum_{j=a+m+1}^{b}\left(\nabla^{p} g(j)\right)\right] \\
& =\nabla^{p} g(a) \\
& \quad+\frac{1}{(b-a-m) \Gamma(\mu-p)} \sum_{j=a+m+1}^{b}\left(\sum_{\tau=a+1}^{j}(j-\tau+1)^{\overline{\mu-p-1}}\left(\nabla_{(a+1) *}^{\mu} g(\tau)\right)\right) .
\end{aligned}
$$

Multiplying the above two terms, we get

$$
\begin{aligned}
& \frac{1}{(b-a-m)^{2}}\left[\sum_{j=a+m+1}^{b}\left(\nabla^{p} f(j)\right)\right]\left[\sum_{j=a+m+1}^{b}\left(\nabla^{p} g(j)\right)\right] \\
& =\left(\nabla^{p} f(a)\right)\left(\nabla^{p} g(a)\right) \\
& +\frac{1}{(b-a-m)^{2}[\Gamma(\mu-p)]^{2}}\left[\sum_{j=a+m+1}^{b}\left(\sum_{\tau=a+1}^{j}(j-\tau+1)^{\overline{\mu-p-1}}\left(\nabla_{(a+1) *}^{\mu} f(\tau)\right)\right)\right]
\end{aligned}
$$




$$
\begin{aligned}
& \cdot\left[\sum_{j=a+m+1}^{b}\left(\sum_{\tau=a+1}^{j}(j-\tau+1)^{\overline{\mu-p-1}}\left(\nabla_{(a+1) *}^{\mu} g(\tau)\right)\right)\right] \\
& +\frac{\left(\nabla^{p} f(a)\right)}{(b-a-m) \Gamma(\mu-p)}\left[\sum_{j=a+m+1}^{b}\left(\sum_{\tau=a+1}^{j}(j-\tau+1)^{\overline{\mu-p-1}}\left(\nabla_{(a+1) *}^{\mu} g(\tau)\right)\right)\right] \\
& +\frac{\left(\nabla^{p} g(a)\right)}{(b-a-m) \Gamma(\mu-p)}\left[\sum_{j=a+m+1}^{b}\left(\sum_{\tau=a+1}^{j}(j-\tau+1)^{\overline{\mu-p-1}}\left(\nabla_{(a+1) *}^{\mu} f(\tau)\right)\right)\right] .
\end{aligned}
$$

Using (8) and (9), we obtain

$$
\begin{aligned}
\frac{1}{(b-a-m)} \sum_{j=a+m+1}^{b}\left[\left(\nabla^{p} f(j)\right)\left(\nabla^{p} g(j)\right)\right] & -\frac{1}{(b-a-m)^{2}}\left[\sum_{j=a+m+1}^{b}\left(\nabla^{p} f(j)\right)\right]\left[\sum_{j=a+m+1}^{b}\left(\nabla^{p} g(j)\right)\right] \\
\leq & \frac{M_{1} M_{2}}{(b-a-m)[\Gamma(\mu-p)]^{2}} \sum_{j=a+m+1}^{b}\left[\sum_{\tau=a+1}^{j}(j-\tau+1)^{\overline{\mu-p-1}}\right]\left[\sum_{\tau=a+1}^{j}(j-\tau+1)^{\overline{\mu-p-1}}\right] \\
& -\frac{m_{1} m_{2}}{(b-a-m)^{2}[\Gamma(\mu-p)]^{2}}\left[\sum_{j=a+m+1}^{b}\left[\sum_{\tau=a+1}^{j}(j-\tau+1)^{\overline{\mu-p-1}}\right]\right] \\
& \cdot\left[\sum_{j=a+m+1}^{b}\left[\sum_{\tau=a+1}^{j}(j-\tau+1)^{\overline{\mu-p-1}}\right]\right] \\
= & \frac{M_{1} M_{2}}{(b-a-m)[\Gamma(\mu-p)]^{2}} \sum_{j=a+m+1}^{b}\left[\sum_{\tau=a+1}^{j}(j-\tau+1)^{\overline{\mu-p-1}}\right]^{2} \\
& -\frac{m_{1} m_{2}}{(b-a-m)^{2}[\Gamma(\mu-p)]^{2}}\left[\sum_{j=a+m+1}^{b}\left[\sum_{\tau=a+1}^{j}(j-\tau+1)^{\overline{\mu-p-1}}\right]\right]^{2} .
\end{aligned}
$$

Next, in order to calculate the last two sums, we first observe that

$$
\begin{aligned}
\sum_{\tau=a+1}^{j}(j-\tau+1)^{\overline{\mu-p-1}} & =\int_{a}^{j}(j-\tau+1)^{\overline{\mu-p-1}} \nabla \tau \\
& =\frac{1}{\mu-p}\left[\frac{\Gamma(j-a+2+\mu-p)}{\Gamma(j-a+2)}-\Gamma(\mu-p+2)\right] .
\end{aligned}
$$

Therefore,

$$
\begin{aligned}
& \sum_{j=a+m+1}^{b}\left[\sum_{\tau=a+1}^{j}(j-\tau+1)^{\overline{\mu-p-1}}\right] \\
& =\frac{1}{\mu-p} \sum_{j=a+m+1}^{b}\left[\frac{\Gamma(j-a+2+\mu-p)}{\Gamma(j-a+2)}-\Gamma(\mu-p+2)\right] \\
& =\frac{1}{\mu-p}\left[\frac{\Gamma(m+3+\mu-p)}{\Gamma(m+3)}+\cdots+\frac{\Gamma(b-a+2+\mu-p)}{\Gamma(b-a+2)}\right]-\frac{b-a-m}{\mu-p} \Gamma(\mu-p+2)
\end{aligned}
$$




$$
\begin{aligned}
= & \frac{1}{\mu-p}\left[(m+3)^{\overline{\mu-p}}+(m+4)^{\overline{\mu-p}}+\cdots+(b-a+2)^{\overline{\mu-p}}\right] \\
& -\frac{b-a-m}{\mu-p} \Gamma(\mu-p+2) \\
= & \frac{1}{\mu-p} \sum_{s=m+3}^{b-a+2}(s)^{\overline{\mu-p}}-\frac{b-a-m}{\mu-p} \Gamma(\mu-p+2) \\
= & \frac{1}{\mu-p} \int_{m+2}^{b-a+2} \tau^{\overline{\mu-p}} \nabla \tau-\frac{b-a-m}{\mu-p} \Gamma(\mu-p+2) \\
= & \frac{1}{\mu-p}\left[\frac{(b-a+1)^{\overline{\mu-p+1}}-(m+1)^{\overline{\mu-p+1}}}{\mu-p+1}\right]-\frac{b-a-m}{\mu-p} \Gamma(\mu-p+2) .
\end{aligned}
$$

Similarly, we get

$$
\begin{aligned}
& \sum_{j=a+m+1}^{b}\left[\sum_{\tau=a+1}^{j}(j-\tau+1)^{\overline{\mu-p-1}}\right]^{2} \\
& \leq \frac{1}{(\mu-p)^{2}}\left[\sum_{j=a+m+1}^{b}(j-\tau+2)^{\overline{\mu-p}}\right]^{2}+\frac{1}{(\mu-p)^{2}}\left[2^{\overline{\mu-p}}\right]^{2}(b-a-m),
\end{aligned}
$$

which by use of Hölder's inequality transforms into

$$
\begin{aligned}
& \sum_{j=a+m+1}^{b}\left[\sum_{\tau=a+1}^{j}(j-\tau+1)^{\overline{\mu-p-1}}\right]^{2} \\
& \leq \frac{(b-a-m+4)^{1 / 2}}{(\mu-p)^{2}}\left(\sum_{s=m+3}^{b-a+2}\left[(s)^{\overline{\mu-p}}\right]^{4}\right)^{1 / 2}+\frac{b-a-m}{(\mu-p)^{2}}\left[2^{\overline{\mu-p}}\right]^{2} .
\end{aligned}
$$

By hypothesis,

$$
\sum_{j=a+m+1}^{b}\left[\sum_{\tau=a+1}^{j}(j-\tau+1)^{\overline{\mu-p-1}}\right]^{2} \leq \frac{Q(b-a-m+4)^{1 / 2}}{(\mu-p)^{2}}+\frac{(b-a-m)\left[2^{\overline{\mu-p}}\right]^{2}}{(\mu-p)^{2}}
$$

Consequently, we get

$$
\begin{aligned}
& \frac{1}{b-a-m} \sum_{j=a+m+1}^{b}\left[\left(\nabla^{p} f(j)\right)\left(\nabla^{p} g(j)\right)\right] \\
& \quad-\frac{1}{(b-a-m)^{2}}\left[\sum_{j=a+m+1}^{b} \nabla^{p} f(j)\right]\left[\sum_{j=a+m+1}^{b} \nabla^{p} g(j)\right] \\
& \leq \frac{M_{1} M_{2} C_{1}-m_{1} m_{2} C_{2}}{(b-a-m)^{2}[\Gamma(\mu-p+1)]^{2}} .
\end{aligned}
$$

Example Let $\mu=3.5, m=4, p=2, b=7, a=1$ in Theorem 2.1. Define

$$
f(t)=g(t)=(t-1)^{\overline{4}}
$$


where $t \in\{-2,-1,0,1, \ldots, 7\}$. Here $j \in\{5,6,7\}$. Using Theorem 2.1, we obtain

$$
\begin{aligned}
& \frac{1}{2} \sum_{t=6}^{7}\left(\nabla^{2}(t-1)^{\overline{4}}\right)\left(\nabla^{2}(t-1)^{\overline{4}}\right)-\frac{1}{4}\left[\sum_{t=6}^{7}\left(\nabla^{2}(t-1)^{\overline{4}}\right)\right]\left[\sum_{t=6}^{7}\left(\nabla^{2}(t-1)^{\overline{4}}\right)\right] \\
& \quad \leq \frac{M_{1} M_{2} C_{1}-m_{1} m_{2} C_{2}}{4[\Gamma(2.5)]^{2}}
\end{aligned}
$$

where $M_{1}=36, M_{2}=37, m_{1}=15, m_{2}=20$ and $C_{1}=3,362, C_{2}=6,572$, and $Q=676.92$.

\section{Competing interests}

The authors declare that they have no competing interests.

\section{Authors' contributions}

AFG conceived the study, and participated in its design and coordination. BK carried out the mathematical studies and participated in the sequence alignment and drafted the manuscript. ACP participated in the design of the study. KT conceived the study, and participated in its design and coordination. All authors read and approved the final manuscript.

\section{Author details}

${ }^{1}$ Department of Mathematics, Faculty of Science, Ankara University, Ankara, Turkey. ${ }^{2}$ Department of Mathematics and Computer Science, Çankaya University, Ankara, 06810, Turkey. ${ }^{3}$ Department of Mathematics, University of Nebraska-Lincoln, Lincoln, NE 68588-0130, USA.

\section{Received: 20 February 2013 Accepted: 24 January 2014 Published: 20 Feb 2014}

\section{References}

1. Boros, G, Moll, V: Irresistible Integrals: Symbols, Analysis and Experiments in the Evaluation of Integrals. Cambridge University Press, Cambridge (2004)

2. Cerone, P, Dragomir, SS: A refinement of the Grüss inequality and applications. Tamkang J. Math. 38, 37-49 (2007)

3. Grüss, G: Über das Maximum des absoluten Betrages von $\frac{1}{b-a} \int_{a}^{b} f(x) g(x) d x-\frac{1}{(b-a)^{2}} \int_{a}^{b} f(x) d x \int_{a}^{b} g(x) d x$. Math. Z. 39, 215-226 (1935)

4. Mercer, AMD: An improvement of the Grüss inequality. JIPAM. J. Inequal. Pure Appl. Math. 6(4), Article ID 93 (2005) (electronic)

5. Mitrinović, DS, Pečarić, JE, Fink, AM: Classical and New Inequalities in Analysis. Kluwer Acadademic, Dordrecht (1993)

6. Anastassiou, GA: Multivariate Fink type identity and multivariate Ostrowski, comparison of means and Grüss type inequalities. Math. Comput. Model. 46, 351-374 (2007)

7. Bohner, M, Matthews, T: The Grüss inequality on time scales. Commun. Math. Anal. 3, 1-8 (2007) (electronic)

8. Dragomir, SS: A Grüss type discrete inequality in inner product spaces and applications. J. Math. Anal. Appl. 250 494-511(2000)

9. Liu, Z: Notes on a Grüss type inequality and its applications. Vietnam J. Math. 35, 121-127 (2007)

10. Perić, I, Rajić, R: Grüss inequality for completely bounded maps. Linear Algebra Appl. 390, 292-298 (2004)

11. Pečarić, JE, Tepeš, B: On the Grüss type inequalities of Dragomir and Fedotov. J. Inequal. Pure Appl. Math. 4(5), Article ID 91 (2003) (electronic)

12. Pečarić, JE, Tepeš, B: A note on Grüss type inequality in terms of $\Delta$-seminorms. Pril. - Maked. Akad. Nauk. Umet., Odd. Mat.-Teh. Nauki 23/24, 29-35 (2004)

13. Goodrich, CS: Continuity of solutions to discrete fractional initial value problems. Comput. Math. Appl. 59, 3489-3499 (2010)

14. Goodrich, CS: Existence and uniqueness of solutions to a fractional difference equation with nonlocal conditions. Comput. Math. Appl. 61, 191-202 (2011)

15. Goodrich, CS: On discrete sequential fractional boundary value problems. J. Math. Anal. Appl. 385, 111-124 (2012)

16. Abdeljawad, T, Atici, FM: On the definitions of nabla fractional operators. Abstr. Appl. Anal. 2012, Article ID 406757, Special Issue (2012)

17. Anderson, DR: Taylor polynomials for nabla dynamic equations on time scales. Panam. Math. J. 12, 17-27 (2002)

18. Anastassiou, GA: Nabla discrete fractional calculus and nabla inequalities. Math. Comput. Model. 51, 562-571 (2010)

10.1186/1029-242X-2014-86

Cite this article as: Güvenilir et al.: Nabla discrete fractional Grüss type inequality. Journal of Inequalities and Applications 2014, 2014:86 